\title{
Acute Disseminated Encephalomyelitis
}

National Institute of Neurological Disorders and Stroke (NINDS)

\section{Source}

National Institute of Neurological Disorders and Stroke (NINDS). Acute Disseminated

Encephalomyelitis Information Page.

Acute disseminated encephalomyelitis (ADEM) is characterized by a brief but widespread attack of inflammation in the brain and spinal cord that damages myelin - the protective covering of nerve fibers. ADEM often follows viral or bacterial infections, or less often, vaccination for measles, mumps, or rubella. The symptoms of ADEM appear rapidly, beginning with encephalitis-like symptoms such as fever, fatigue, headache, nausea and vomiting, and in the most severe cases, seizures and coma. ADEM typically damages white matter (brain tissue that takes its name from the white color of myelin), leading to neurological symptoms such as visual loss (due to inflammation of the optic nerve) in one or both eyes, weakness even to the point of paralysis, and difficulty coordinating voluntary muscle movements (such as those used in walking). ADEM is sometimes misdiagnosed as a severe first attack of multiple sclerosis (MS), since the symptoms and the appearance of the white matter injury on brain imaging may be similar. However, ADEM has several features which differentiate it from MS. First, unlike MS patients, persons with ADEM will have rapid onset of fever, a history of recent infection or immunization, and some degree of impairment of consciousness, perhaps even coma; these features are not typically seen in MS. Children are more likely than adults to have ADEM, whereas MS is a rare diagnosis in children. In addition, ADEM usually consists of a single episode or attack of widespread myelin damage, while MS features many attacks over the course of time. Doctors will often use imaging techniques, such as MRI (magnetic resonance imaging), to search for old and new lesions (areas of damage) on the brain. The presence of older brain lesions on MRI suggest that the condition may be MS rather than ADEM, since MS can cause brain lesions before symptoms become obvious. In rare situations, a brain biopsy may be necessary to differentiate between ADEM and some other diseases that involve inflammation and damage to myelin. 